\title{
Stabilization for Sampled-Data Systems under Noisy Sampling Interval *
}

\author{
Bo Shen ${ }^{\mathrm{a}, *}$, Zidong Wang ${ }^{\mathrm{b}}$, Tingwen Huang ${ }^{\mathrm{c}}$ \\ ${ }^{a}$ School of Information Science and Technology, Donghua University, Shanghai 200051, China. \\ ${ }^{\mathrm{b}}$ Department of Information Systems and Computing, Brunel University, Uxbridge, Middlesex, UB8 3PH, U.K. \\ ${ }^{\mathrm{c}}$ Department of Mathematics, Texas A\&M University at Qatar, Doha, P.O.Box 23874, Qatar.
}

\begin{abstract}
In engineering practice, the sampling interval for a sampled-data system often fluctuates around a nominal/ideal value based on certain probability distributions that can be specified a priori through statistical tests. In this paper, a fundamental stabilization problem is investigated for a class of sampled-data systems under noisy sampling interval. The stochastic sampleddata control system under consideration is first converted into a discrete-time system whose system matrix is represented as an equivalent yet tractable form via the matrix exponential computation. Then, by introducing a Vandermonde matrix, the mathematical expectation of the quadratic form of the system matrix is computed. By recurring to the Kronecker product operation, the sampled-data stabilization controller is designed such that the closed-loop system is stochastically stable in the presence of noisy sampling interval. Subsequently, a special case is considered where the sampling interval obeys the continuous uniform distribution and the corresponding stabilization controller is designed. Finally, a numerical simulation example is provided to demonstrate the effectiveness of the proposed design approach.
\end{abstract}

Key words: Matrix exponential; noisy sampling interval; sampled-data control systems; stochastic control; synthesis of stochastic systems.

\section{Introduction}

The past few decades have witnessed constant research interests on both the analysis and synthesis problems of sampled-data control systems, and a rich body of literature has been available, see e.g. $[2,9,16,18]$ and the references therein. Traditionally, the sampling interval has been assumed to be constant in a periodic sampled-data system. Such an assumption is, however, not always true in practical engineering. For example, in networked and embedded control systems, it is often the case that the sampling intervals are uncertain and/or vary with time due largely to unpredictable network-induced phenomena. Recently, the aperiodic sampling issue has stirred a great deal of research attention in the design and control of sampled-data systems. In [6,13], an input delay approach has been proposed to deal with the uncertain sampling intervals and the aperiodic sampled-data control problems have been investigated in virtue of the Lyapunov-Krasovskii theorem. In $[1,11,17]$, the aperiodic sampled-data control system has been transformed into a discrete-time system by using the exact integra-

\footnotetext{
* This work was supported in part by the National Natural Science Foundation of China under Grants 61473076 and 61329301, the Shu Guang project of Shanghai Municipal Education Commission and Shanghai Education Development Foundation under Grant 13SG34, the Program for Professor of Special Appointment (Eastern Scholar) at Shanghai Institutions of Higher Learning, the Fundamental Research Funds for the Central Universities, the DHU Distinguished Young Professor Program, and the National Priority Research Project NPRP 4-1162-1-181 funded by Qatar National Research Fund.

* Corresponding author. Tel.: +86 21 67792371-811.

Email address: bo.shen@dhu.edu.cn (Bo Shen).
}

tion over a sampling interval and less conservative stability criteria have been derived.

It should be pointed out that, in most existing literature, the aperiodic sampling intervals have been implicitly assumed to be deterministic. Nevertheless, it is quite common in practice that the aperiodic samplings often occur in a probabilistic way owing to some undesirable physical constraints such as aperiodic faults in the samplers, fluctuated network loads, intermittent signal quantization/saturation and unwanted changes of some components of the system itself. A typical example is the seismic data extraction where the sampleddata is collected from the random interspersed observation points along the trajectory of the process. Recently, the sampled-data control problem under noisy sampling intervals has begun to receive some initial attention and the corresponding results have been scattered. In [7], the sampled-data $H_{\infty}$ control problem has been investigated when sampling intervals randomly switch between two given values and, in [8], the sampled-data $\mathrm{H}_{2}$-optimal controller has been designed with sampling intervals obeying the Erlang distribution. Note that the sampling models in $[7,8]$, though interesting, are quite special and there appears to be a need to cater for more general aperiodic sampling phenomena. As such, it is our first motivation in this paper to establish a fairly comprehensive aperiodic sampling model and examine how the aperiodic sampling interval impacts on the stability and performance of the overall sampled-data system.

In order to obtain the stability criteria for the sampleddata control system with less conservativeness, a preferred approach would be to transform the underlying controlled system into its equivalent discrete-time counterpart. In this case, the resulting discrete-time closedloop system is highly nonlinear with respect to the sam- 
pling interval which, in turn, gives rise to significant difficulties in the analysis and design of the sampled-data control systems. More specifically, three fundamental difficulties are identified as follows: 1) how can we find an equivalent yet tractable representation for the system matrix which includes a matrix exponential and its integration simultaneously over the sampling interval? 2) how can we compute the mathematical expectation of the quadratic form of a matrix exponential involving random scalars? and 3) in the design of stochastic sampled-data control system, how can we derive an easy-to-implement design algorithm according to the established stability criteria? It is, therefore, our second yet primary motivation in our paper to offer satisfactory answers to the three questions.

In response to the above discussion, in this paper, we aim to investigate the stabilization problem for sampleddata systems with noisy sampling interval. The main contributions of this paper are summarized as follows: 1) a new stabilization control problem is addressed for a class of sampled-data systems where the sampling intervals fluctuate around a nominal/ideal value according to certain probability distributions; 2) by employing the matrix theory such as matrix exponential computation, Vandermonde matrix and Kronecker product operation, the stabilization controller is designed; and 3) a special case is considered where the sampling intervals obey the continuous uniform distribution. Finally, a numerical simulation example is presented to show the effectiveness of the proposed sampled-data control scheme.

\section{Problem Formulation}

Consider the following continuous-time system

$$
\dot{x}(t)=A x(t)+B u(t)
$$

where $x(t) \in \mathbb{R}^{n}$ is the state vector, $u(t) \in \mathbb{R}^{m}$ is the control input, $A$ is the system matrix and $B$ is the input matrix. The initial value is given by $x_{0}$.

The control input $u(t)$ is generated by a zero-order hold function with a sequence of hold times $0=t_{0}<t_{1}<$ $\cdots<t_{k}<\cdots$

$$
u(t)=K x\left(t_{k}\right), \quad t_{k} \leq t<t_{k+1}
$$

where $K$ is the gain matrix to be determined and $t_{k}$ denotes the sampling instant satisfying $\lim _{k \rightarrow \infty} t_{k}=\infty$. By substituting (2) into (1), the closed-loop system is obtained as follows

$$
\dot{x}(t)=A x(t)+B K x\left(t_{k}\right), \quad t_{k} \leq t<t_{k+1} .
$$

Integrating the above equation from $t_{k}$ to $t_{k+1}$, one has

$$
\begin{aligned}
x\left(t_{k+1}\right)= & \left(\mathrm{e}^{A\left(t_{k+1}-t_{k}\right)}\right. \\
& \left.\quad+\int_{t_{k}}^{t_{k+1}} \mathrm{e}^{A\left(t_{k+1}-\theta\right)} d \theta B K\right) x\left(t_{k}\right) .
\end{aligned}
$$

Letting the sampling interval be $T_{k}=t_{k+1}-t_{k}$ and denoting $x\left(t_{k}\right)$ by $x_{k}$, we come up with a discrete-time system of the following form

$$
x_{k+1}=\left(\mathrm{e}^{A T_{k}}+\int_{0}^{T_{k}} \mathrm{e}^{A s} d s B K\right) x_{k} .
$$

The sampling interval $T_{k}$ under consideration is subject to noisy perturbations and consists of two parts, i.e., $T_{k}=T+v_{k}$ where $T$ is a constant which stands for the nominal sampling interval and $v_{k}$ is a random variable which accounts for the sampling errors/drifts/deviations resulting from unpredictable environmental phenomena. The probability density function of the random variable $v_{k}$ is denoted by $f(v)$ where the argument $v$ satisfies $T+v>0$.

Remark 1 It is worth mentioning that, to date, very little attention has been paid to noisy sampling interval issue despite its practical significance in engineering. In [7, 8], the noisy sampling interval has been taken into account where the sampling intervals have been simply assumed to obey Bernoulli or Erlang distribution. In this paper, the probability distributions of the sampling intervals are general that include the above probability distributions as special cases and the aim of this paper is to develop a design approach for the sampled-data control systems with such a general noisy sampling model.

Note that the system (5) is a discrete-time stochastic system due to the random nature of the sampling error, and therefore the following notion of stochastic stability is needed.

Definition 1 [5] The discrete-time stochastic system (5) is said to be stochastically stable if

$$
\mathbb{E}\left\{\sum_{k=0}^{\infty}\left\|x_{k}\right\|^{2}\right\}<\infty .
$$

In this paper, we aim to investigate the sampled-data stabilization problem for the system (1) in presence of the random sampling error, that is, we are interested in finding the controller gain matrix $K$ such that the discrete-time stochastic system (5) is stochastically stable with respect to noisy sampling intervals obeying certain probability distributions.

\section{Main Results}

To start with, a sufficient condition is provided in the following well-known lemma for the stochastic stability of the system (5).

Lemma 1 Given the controller gain matrices $K$, the $s$ tochastic system (5) is stochastically stable if there exists a positive definite matrix $Q$ such that the following inequality holds:

$$
\begin{aligned}
\mathbb{E}\left\{\left(e^{A T_{k}}\right.\right. & \left.+\int_{0}^{T_{k}} e^{A s} d s B K\right)^{T} Q \\
& \left.\times\left(e^{A T_{k}}+\int_{0}^{T_{k}} e^{A s} d s B K\right)\right\}-Q<0 .
\end{aligned}
$$

Proof: The proof of this lemma is straightforward and is therefore omitted.

It can be seen from (7) that the random variable $T_{k}$ appears in the upper boundary of integral, and this makes it difficult to directly calculate the mathematical expectation of the integral. In what follows, our main efforts will be made towards the computation of this mathematical expectation by recurring to the matrix theory.

Firstly, it follows from Theorem 1 in [14] that 


$$
\mathrm{e}^{C T_{k}}=\left[\begin{array}{cc}
\mathrm{e}^{A T_{k}} & \int_{0}^{T_{k}} \mathrm{e}^{A s} d s B \\
0 & I
\end{array}\right]
$$

where

$$
C=\left[\begin{array}{cc}
A & B \\
0 & 0
\end{array}\right] .
$$

Then, $\mathrm{e}^{A T_{k}}+\int_{0}^{T_{k}} \mathrm{e}^{A s} d s B K$ can be rewritten as

$$
\begin{aligned}
& \mathrm{e}^{A T_{k}}+\int_{0}^{T_{k}} \mathrm{e}^{A s} d s B K \\
= & {\left[\begin{array}{ll}
I & 0
\end{array}\right]\left[\begin{array}{cc}
\mathrm{e}^{A T_{k}} & \int_{0}^{T_{k}} \mathrm{e}^{A s} d s B \\
0 & I
\end{array}\right]\left[\begin{array}{c}
I \\
K
\end{array}\right] } \\
= & {\left[\begin{array}{ll}
I & 0
\end{array}\right] \mathrm{e}^{C T} \mathrm{e}^{C v_{k}}\left[\begin{array}{c}
I \\
K
\end{array}\right] . }
\end{aligned}
$$

Secondly, we transform the matrix exponential $\mathrm{e}^{C v_{k}}$ into a tractable form. In order to avoid unnecessary mathematical complexity, we make the following assumption.

Assumption 1 Suppose that the matrix A has different eigenvalues $\lambda_{i} \neq 0(i=1,2, \cdots, n)$ and the control input $u(t)$ is a scalar, i.e., $m=1$.

Under Assumption 1, it is easily known that the eigenvalues of matrix $C$ (denoted by $\left.\lambda_{C, i}\right)$ are $\lambda_{C, i}=\lambda_{i}$ $(i=1,2, \cdots, n)$ and $\lambda_{C, n+1}=0$. Then, the Vandermonde matrix generated by the eigenvalues of matrix $C$ is given by

$$
V=\left[\begin{array}{cccc}
1 & 1 & \cdots & 1 \\
\lambda_{C, 1} & \lambda_{C, 2} & \cdots & \lambda_{C, n+1} \\
\vdots & \vdots & \ddots & \vdots \\
\lambda_{C, 1}^{n} & \lambda_{C, 2}^{n} & \cdots & \lambda_{C, n+1}^{n}
\end{array}\right] .
$$

By following the same analysis in [10], the matrix exponential $\mathrm{e}^{C v_{k}}$ can be expressed by

$$
\mathrm{e}^{C v_{k}}=\sum_{i=1}^{n+1} \mathrm{e}^{\lambda_{C, i} v_{k}} C_{i}
$$

where

$$
C_{i}=\sum_{j=1}^{n+1} \nu_{i j} C^{j-1}
$$

and $\nu_{i j}$ is the $(i, j)$ entry of $V^{-1}$.

Remark 2 Under Assumption 1, it is ensured that the matrix $C$ has different eigenvalues. In this case, the matrix exponential $e^{C v_{k}}$ can be computed according to (12) and (13). In case the matrix $C$ has repeated eigenvalues such as the multi-input case, similar formulas can be obtained by introducing an appropriate confluent Vandermonde matrix.

Introduce the matrix $\Pi_{f}$ defined by

$$
\Pi_{f}=\left[\pi_{i j}^{f}\right]_{(n+1) \times(n+1)}
$$

with

$$
\pi_{i j}^{f}=\left\{\begin{array}{cc}
\int_{-\infty}^{+\infty} \mathrm{e}^{2 \lambda_{C, i} v} f(v) d v, & i=j, \\
\int_{-\infty}^{+\infty} \mathrm{e}^{\left(\lambda_{C, i}+\lambda_{C, j}\right) v} f(v) d v, & i \neq j .
\end{array}\right.
$$

Lemma 2 For the matrix $\Pi_{f}$ defined in (14), there exists a matrix $U_{f}$ such that $\Pi_{f}=U_{f}^{T} U_{f}$.

Proof: From (14), it is easily seen that the matrix $\Pi_{f}$ is symmetric. For any $\eta \neq 0 \in \mathbb{R}^{n+1}$, we have

$$
\eta^{T} \Pi_{f} \eta=\int_{-\infty}^{+\infty} \eta^{T} \Pi_{v} \eta f(v) d v
$$

where $\Pi_{v}=\left[\pi_{i j}^{v}\right]_{(n+1) \times(n+1)}$ with

$$
\pi_{i j}^{v}=\left\{\begin{array}{cc}
\mathrm{e}^{2 \lambda_{C, i} v}, & i=j, \\
\mathrm{e}^{\left(\lambda_{C, i}+\lambda_{C, j}\right) v}, & i \neq j .
\end{array}\right.
$$

Note that, for any $v \in \mathbb{R}$, the matrix $\Pi_{v}$ defined in (16) is always positive semi-definite and, therefore, for al$l \eta \in \mathbb{R}^{n+1}$, we have $\eta^{T} \Pi_{v} \eta \geq 0$. By considering that the probability density function $f(v)$ is positive for all $v \in \mathbb{R}$, it follows from (15) that $\eta^{T} \Pi_{f} \eta \geq 0$ for all $\eta \in \mathbb{R}^{n+1}$. This implies that the matrix $\Pi_{f}$ is also positive semidefinite and hence we can find a matrix $U_{f}$ such that $\Pi_{f}=U_{f}^{T} U_{f}$. The proof of Lemma 2 is complete.

Now, our main results are given as follows.

Theorem 1 For the continuous-time system (1) with the sampled-data controller given by (2), the sampleddata stabilization problem with noisy sampling interval is solvable if there exist matrices $P>0$ and $Y$ such that the following inequality holds:

$$
\left[\begin{array}{cc}
-P & \Phi_{f} \\
* & -(I \otimes P)
\end{array}\right]<0
$$

where

$$
\begin{aligned}
\Phi_{f} & =\left[\begin{array}{ll}
P & Y^{T}
\end{array}\right] e^{C^{T} T} \bar{C}^{T}\left(U_{f}^{T} \otimes\left[\begin{array}{l}
I \\
0
\end{array}\right]\right), \\
\bar{C} & =\left[\begin{array}{llll}
C_{1}^{T} & C_{2}^{T} & \cdots & C_{n+1}^{T}
\end{array}\right]^{T} .
\end{aligned}
$$

Furthermore, if inequality (17) is feasible, the desired controller gain is given by

$$
K=Y P^{-1} \text {. }
$$

Proof: According to (12), one has

$$
\begin{aligned}
& \mathbb{E}\left\{\mathrm{e}^{C^{T} v_{k}}\left[\begin{array}{l}
I \\
0
\end{array}\right] Q\left[\begin{array}{ll}
I & 0
\end{array}\right] \mathrm{e}^{C v_{k}}\right\} \\
= & \mathbb{E}\left\{\left(\sum_{i=1}^{n+1} \mathrm{e}^{\lambda_{C, i} v_{k}} C_{i}\right)^{T}\left[\begin{array}{l}
I \\
0
\end{array}\right]\right. \\
& \left.\times Q\left[\begin{array}{ll}
I & 0
\end{array}\right] \sum_{i=1}^{n+1} \mathrm{e}^{\lambda_{C, i} v_{k}} C_{i}\right\} \\
= & \bar{C}^{T}\left(\left(\int_{-\infty}^{+\infty} \Pi_{v} f(v) d v\right) \otimes\left(\left[\begin{array}{l}
I \\
0
\end{array}\right] Q\left[\begin{array}{ll}
I & 0
\end{array}\right]\right)\right) \bar{C} \\
= & \bar{C}^{T}\left(\Pi_{f} \otimes\left(\left[\begin{array}{l}
I \\
0
\end{array}\right] Q\left[\begin{array}{ll}
I & 0
\end{array}\right]\right)\right) \bar{C} \\
= & \bar{C}^{T}\left(U_{f}^{T} \otimes\left[\begin{array}{l}
I \\
0
\end{array}\right]\right)(I \otimes Q)\left(U_{f} \otimes\left[\begin{array}{ll}
I & 0
\end{array}\right]\right) \bar{C}
\end{aligned}
$$


where $\Pi_{f}$ and $\Pi_{v}$ are defined in (14) and (16), respectively. It then follows from (10) that

$$
\begin{aligned}
& \mathbb{E}\left\{\left(\mathrm{e}^{A T_{k}}+\int_{0}^{T_{k}} \mathrm{e}^{A s} d s B K\right)^{T} Q\right. \\
& \left.\times\left(\mathrm{e}^{A T_{k}}+\int_{0}^{T_{k}} \mathrm{e}^{A s} d s B K\right)\right\} \\
= & {\left[\begin{array}{ll}
I & K^{T}
\end{array}\right] \mathrm{e}^{C^{T} T} \mathbb{E}\left\{\mathrm{e}^{C^{T} v_{k}}\left[\begin{array}{l}
I \\
0
\end{array}\right] Q\left[\begin{array}{ll}
I & 0
\end{array}\right] \mathrm{e}^{C v_{k}}\right\} } \\
& \times \mathrm{e}^{C T}\left[\begin{array}{l}
I \\
K
\end{array}\right] \\
= & \bar{\Phi}_{f}(I \otimes Q) \bar{\Phi}_{f}^{T}
\end{aligned}
$$

where $\bar{\Phi}_{f}=\left[\begin{array}{ll}I & K^{T}\end{array}\right] \mathrm{e}^{C^{T} T} \bar{C}^{T}\left(U_{f}^{T} \otimes\left[\begin{array}{l}I \\ 0\end{array}\right]\right)$.

Subsequently, by using the Schur Complement Lemma, the condition (7) in Lemma 1 is true if and only if

$$
\left[\begin{array}{cc}
-Q & \bar{\Phi}_{f}(I \otimes Q) \\
* & -(I \otimes Q)
\end{array}\right]<0
$$

which is equivalent to inequality (17) by performing a congruence transformation of $\operatorname{diag}\left\{Q^{-1}, I \otimes Q^{-1}\right\}$ and noting the relations $P=Q^{-1}$ and $Y=K P$. That is, inequality (17) implies that condition (7) in Lemma 1 holds, and the rest of the proof follows directly from Lemma 1.

In Theorem 1, the existence condition of the sampleddata controller is obtained and, if the probability density function of the sampling error is determined, the desired sampled-data controller can be designed by finding a solution to inequality (17). The feature of the design approach developed is that we have exploited the matrix theories including matrix exponential computation, Vandermonde matrix and Kronecker product operation which deal well with the difficulties from both the random nature and the nonlinear characteristics.

Remark 3 Note that the design approach developed can cope with the case when the sampling intervals follow certain probability distributions. For other sampling disturbance, so long as the probability distributions of their sampling intervals are known, the design approach developed is always effective.

In what follows, we discuss a special case where the sampling error obeys the continuous uniform distribution.

Suppose that the sampling error $v_{k}$ follows the continuous uniform distribution between $-\delta$ and $\delta$, i.e, the probability density function of the random variable $v_{k}$ is given by

$$
f(v)=\left\{\begin{array}{cc}
\frac{1}{2 \delta}, & -\delta \leq v \leq \delta \\
0, & \text { elsewhere }
\end{array}\right.
$$

where $0<\delta<T$.

By the same definition of $\Pi_{f}$ as before, we obtain the matrix $\Pi_{\delta}=\left[\pi_{i j}^{\delta}\right]_{(n+1) \times(n+1)}$ with

$$
\pi_{i j}^{\delta}=\left\{\begin{array}{cc}
\lim _{\lambda \rightarrow \lambda_{C, i}} \frac{1}{4 \delta \lambda}\left(\mathrm{e}^{2 \lambda \delta}-\mathrm{e}^{-2 \lambda \delta}\right), & i=j, \\
\lim _{\lambda \rightarrow\left(\lambda_{C, i}+\lambda_{C, j}\right)} \frac{1}{2 \delta \lambda}\left(\mathrm{e}^{\lambda \delta}-\mathrm{e}^{-\lambda \delta}\right), & i \neq j .
\end{array}\right.
$$

In virtue of Lemma 2, we can find a matrix $U_{\delta}$ such that $\Pi_{\delta}=U_{\delta}^{T} U_{\delta}$. Then, by Theorem 1, the following corollary is easily obtained.

Corollary 1 Suppose that the sampling error $v_{k}$ obeys the continuous uniform distribution between $-\delta$ and $\delta$ $(0<\delta<T)$. If there exist matrices $P>0$ and $Y$ satisfying the following inequality:

$$
\left[\begin{array}{cc}
-P & \Phi_{\delta} \\
* & -(I \otimes P)
\end{array}\right]<0
$$

where

$$
\Phi_{\delta}=\left[\begin{array}{ll}
P & Y^{T}
\end{array}\right] e^{C^{T} T} \bar{C}^{T}\left(U_{\delta}^{T} \otimes\left[\begin{array}{l}
I \\
0
\end{array}\right]\right),
$$

then the sampled-data controller (2) with gain matrix given by (19) achieves the stochastic stability of the stochastic systems (5).

Proof: The proof follows directly from Theorem 1.

Until now, the desired sampled-data stabilization controller has been designed such that the sampled-data control system is stochastically stable when the sampling intervals follow certain probability distributions. As a special case, the sampled-data stabilization controller has been designed when the sampling intervals follow continuous uniform distribution. In next section, an illustrative example shall be given to verify the effectiveness of the proposed design approach.

\section{An Illustrative Example}

In this section, a numerical example is presented to demonstrate the effectiveness of the sampled-data control scheme proposed in this paper.

Consider a continuous-time system described by (1) with the following parameters

$$
A=\left[\begin{array}{cc}
1.8 & -1.3 \\
0 & 1.2
\end{array}\right], \quad B=\left[\begin{array}{c}
1 \\
-1.2
\end{array}\right] .
$$

It is easy to see that the system matrix $A$ has eigenvalues $\lambda_{1}=1.8$ and $\lambda_{2}=1.2$, which implies that the system without control input is unstable.

From the definition of matrix $C$ in (9), it is known that the eigenvalues of matrix $C$ are $\lambda_{C, 1}=1.8, \lambda_{C, 2}=1.2$ and $\lambda_{C, 3}=0$. Then, according to (11) and (13), we have

$$
\begin{aligned}
C_{1} & =\left[\begin{array}{ccc}
1 & -2.1667 \\
0 & 0 & 0 \\
0 & 0 & 0
\end{array}\right], \quad C_{3}=\left[\begin{array}{ccc}
0 & 0 & 0.1667 \\
0 & 0 & 1 \\
0 & 0 & 1
\end{array}\right], \\
C_{2} & =\left[\begin{array}{ccc}
0 & 2.1667 & -2.1667 \\
0 & 1 & -1 \\
0 & 0 & 0
\end{array}\right] .
\end{aligned}
$$


Suppose that the nominal sampling interval is $T=0.8$ and the sampling error $v_{k}$ obeys continuous uniform distribution between -0.3 and 0.3 . By $(24)$, matrix $\Pi_{\delta}$ is obtained as

$$
\Pi_{\delta}=\left[\begin{array}{lll}
1.2061 & 1.1406 & 1.0493 \\
1.1406 & 1.0887 & 1.0217 \\
1.0493 & 1.0217 & 1.0000
\end{array}\right],
$$

from which we can find matrix

$$
U_{\delta}=\left[\begin{array}{ccc}
0.0041 & -0.0063 & 0.0021 \\
0.1334 & 0.0273 & -0.1768 \\
1.0901 & 1.0430 & 0.9843
\end{array}\right]
$$

such that $\Pi_{\delta}=U_{\delta}^{T} U_{\delta}$.

With the above parameters, the inequality (25) can be solved by using the Matlab software and we can obtain a set of feasible solutions as follows

$$
P=\left[\begin{array}{cc}
1.2477 & -1.5093 \\
-1.5093 & 15.2625
\end{array}\right], \quad Y=\left[\begin{array}{ll}
-3.3913 & 19.7073
\end{array}\right] \text {. }
$$

Then, according to (19), the desired controller gain matrix is designed as

$$
K=Y P^{-1}=\left[\begin{array}{ll}
-1.3132 & 1.1614
\end{array}\right] .
$$

In the simulation, the initial value is set as $x_{0}=$ $\left[\begin{array}{ll}1 & 0.8\end{array}\right]^{T}$. Simulation results are presented in Figs. 1-2.

Fig. 1 plots the state trajectories without control input. The state trajectories with sampled-data control input are depicted in Fig. 2 where the asterisks represent the sampling instants. From simulation results, it can be seen that the designed sampled-data controller stabilizes the unstable system very well, which has confirmed that the proposed design approach is effective.

\section{Conclusions}

In this paper, we have studied the stabilization problem for a class of sampled-data systems with noisy sampling interval. The sampling intervals under consideration are uncertain and satisfy certain probability distributions. We have first converted the stochastic sampled-data control system into a discrete-time system. With the help of the matrix theory including matrix exponential computation, Vandermonde matrix and Kronecker product operation, the sampled-data stabilization controller has been designed such that the stochastic sampled-data control system is stochastically stable. Then, we have discussed the design problem of sampled-data controller when the sampling intervals follow the continuous uniform distribution and the corresponding stabilization controller has been designed. Finally, a simulation example has been presented to verify the effectiveness of the proposed design approach. Further research topics include sampled-data control problem under the general noisy sampling interval by taking into account the incomplete information investigated in $[3,4,12,15]$.
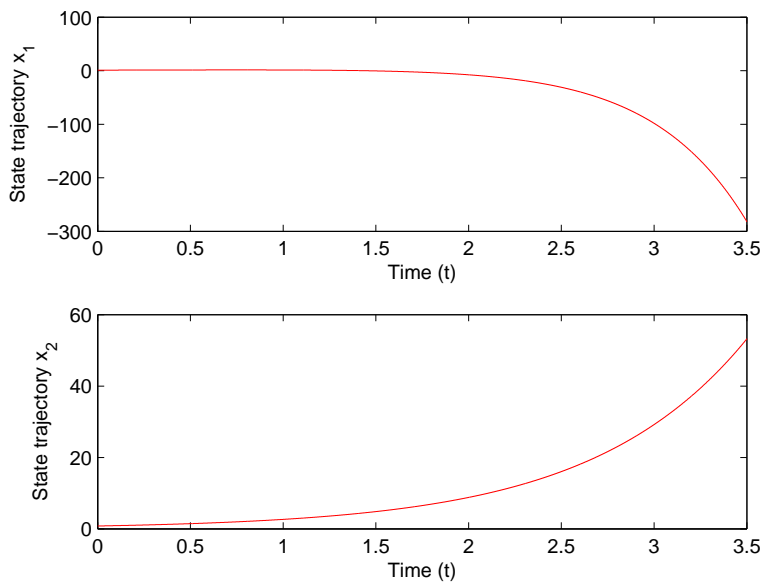

Fig. 1. State trajectories of system without control input
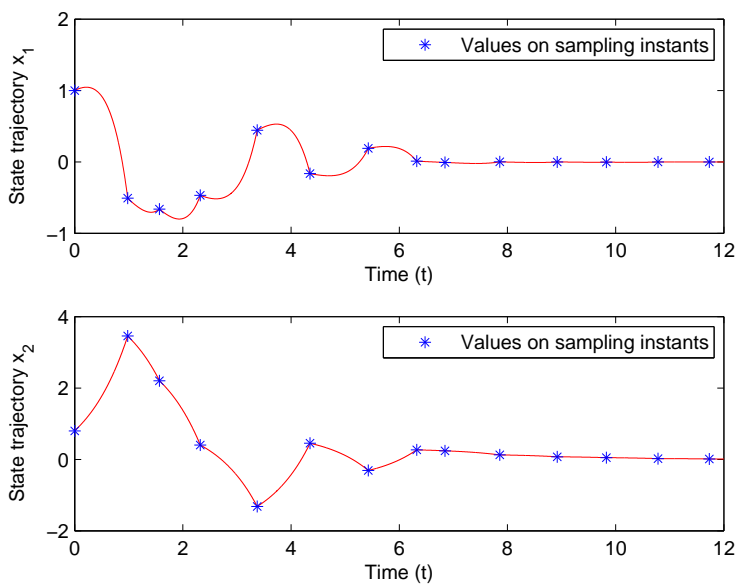

Fig. 2. State trajectories of system with control input

\section{References}

[1] S. Alexandre, A novel stability analysis of linear systems under asynchronous samplings, Automatica, Vol. 48, No. 1, pp. 177-182, Jan. 2012.

[2] D. Dai, T. Hu, A. R. Teel and L. Zaccarian, Output feedback synthesis for sampled-data system with input saturation, Proc. 2010 American Control Conference, pp. 1797-1802, Marriott Waterfront, Baltimore, MD, USA, Jun. 30-Jul. 02, 2010.

[3] D. Ding, Z. Wang, J. Lam and B. Shen, FiniteHorizon $H_{\infty}$ control for discrete time-varying systems with randomly occurring nonlinearities and fading measurements, IEEE Transactions on Automatic Control, in press, DOI:10.1109/TAC.2014.2380671

[4] D. Ding, Z. Wang, B. Shen and H. Dong, Envelopeconstrained $H_{\infty}$ filtering with fading measurements and randomly occurring nonlinearities: the finite horizon case, Automatica, Vol. 55, pp. 37-45, May. 2015.

[5] H. Dong, Z. Wang and H. Gao, Distributed $H_{\infty}$ filtering for a class of Markovian jump nonlinear time-delay systems over lossy sensor networks, Vol. 60, No. 10, pp. 4665-4672, Oct. 2013.

[6] E. Fridman, A. Seuret and J. P. Richard, Robust sampleddata stabilization of linear systems: an input delay approach, Automatica, Vol. 40, No. 8, pp. 1441-1446, Aug. 2004.

[7] H. Gao, J. Wu and P. Shi, Robust sampled-data $H_{\infty}$ control with stochastic sampling, Automatica, Vol. 45, No. 7 , pp. 1729-1736, Jul. 2009. 
[8] A. Kanchanaharuthai and M. Wongsaisuwan, Stochastic $H_{2}-$ optimal controller design for sampled-data systems with random sampled measurement, Proc. 41st SICE Annual Conference, pp. 2042-2047, Osaka, Japan, Aug. 5-7, 2002.

[9] H. K. Lam, Stabilization of nonlinear systems using sampleddata output-feedback fuzzy controller based on polynomialfuzzy-model-based control approach, IEEE Trans. Systems Man and Cybernetics Part B-Cybernetics, Vol. 42, No. 1, pp. 258-267, Feb. 2012.

[10] C. Moler and C. Van Loan, Nineteen dubious ways to compute the exponential of a matrix, twenty-five years later, SIAM Review, Vol. 45, No. 1, pp. 3-49, Mar. 2003.

[11] Y. Oishi and H. Fujioka, Stability and stabilization of aperiodic sampled-data control systems using robust linear matrix inequalities, Automatica, Vol. 46, No. 8, pp. 13271333, Aug. 2010.

[12] B. Shen, A survey on the applications of the Krein-space theory in signal estimation, Systems Science \& Control Engineering: An Open Access Journal, Vol. 2, No. 1, pp. 143149, Jan. 2014.

[13] V. Suplin, E. Fridman and U. Shaked, Sampled-data $H_{\infty}$ control and filtering: Nonuniform uncertain sampling, Automatica, Vol. 43, No. 6, pp. 1072-1083, Jun. 2007.

[14] C. F. Van Loan, Computing integrals involving the matrix exponential, IEEE Trans. Automatic Control, Vol. AC-23, No. 3, pp. 395-404, Jun. 1978.

[15] G. Wei, S. Liu, Y. Song and Y. Liu, Probability-guaranteed set-membership filtering for systems with incomplete measurements, Automatica, Vol. 60, pp. 12-16, 2015.

[16] Y. Xu, H. Su, Y. Pan, Output feedback stabilization for Markov-based nonuniformly sampled-data networked control systems, Systems 83 Control Letters, Vol. 62, No. 8, pp. 656663, Aug. 2013.

[17] S. S. Young, Stability and stabilization of nonuniform sampling systems, Automatica, Vol. 44, No. 12, pp. 32223226, Dec. 2008.

[18] W. Zhang and L. Yu, Stabilization of sampled-data control systems with control inputs missing, IEEE Trans. Automatic Control, Vol. 55, No. 2, pp. 447-452, Feb. 2010. 